\title{
Land Cover Change Detection Based on Genetically Feature Selection and Image Algebra Using Hyperion Hyperspectral Imagery
}

\author{
Seyd Teymoor Seydi, and Mahdi Hasanlou \\ School of Surveying and Geospatial Engineering, \\ College of Engineering, University of Tehran, Tehran, Iran; \\ seydi.teymoor@ut.ac.ir, hasanlou@ut.ac.ir
}

\begin{abstract}
:
The Earth has always been under the influence of population growth and human activities. This process causes the changes in land use. Thus, for optimal management of the use of resources, it is necessary to be aware of these changes. Satellite remote sensing has several advantages for monitoring land use/cover resources, especially for large geographic areas. Change detection and attribution of cultivation area over time present additional challenges for correctly analyzing remote sensing imagery. In this regards, for better identifying change in multi temporal images we use hyperspectral images. Hyperspectral images due to high spectral resolution created special placed in many of field. Nevertheless, selecting suitable and adequate features/bands from this data is crucial for any analysis and especially for the change detection algorithms. This research aims to automatically feature selection for detect land use changes are introduced. In this study, the optimal band images using hyperspectral sensor using Hyperion hyperspectral images by using genetic algorithms and Ratio bands, we select the optimal band. In addition, the results reveal the superiority of the implemented method to extract change map with overall accuracy by a margin of nearly $79 \%$ using multi temporal hyperspectral imagery.
\end{abstract}

Keywords - Hyperspectral, change detection, feature selection, genetic algorithm, Land Cover.

\section{Introduction:}

A world in which we live is constantly changing. With the study can be understand that the origin of these changes: human activities and natural phenomena. This process of change and transformation causes the destruction of some phenomenon, and there are some phenomena and effects. These changes are, for example, the user changes the expansion of cities, soil erosion and natural events that triggered the flooding North of the fields and the sea advance or rivers. Hence, to optimize the management of their resources and exploit the knowledge of this process of change seems to be imperative. Remote sensing (RS) is kind of new source of numerous applications in the field of Earth Sciences that studies one of the most important applications identified changes to the Earth's surface. This process called change detection. This process utilized the differences procedure between the two different phenomenon at the time of measurement. This measuring is vital for better management of the environment $[4,5]$. Nowadays, with the advancement of remote sensing technology, it is possible to obtain spectral band in satellite image format in hundreds of wavelengths. These images are known as hyperspectral images, which can be 
identified with a very high accuracy changes. Most of hyperspectral sensors, acquired using aerial platform. One of most important hyperspectral satellite imagery acquired by Hyperion sensor. Processing such images requires special procedures. The most important issues related to these images is its high spectral dimension, which refer to Hughes phenomena. To solve this problem, different methods are proposed including feature extraction (FE) and feature selection (FS). In the FS method, most suitable bands (features) are selected based on some criteria. The common method for FS is incorporating Optimization Algorithm (OA). In this regards, we can take advantage of the Metaheuristic Optimization Algorithm (MOA). The most frequently asked of these methods is that the nature of the genetic algorithm (GA) based on artificial Intelligence-Inspired. The GA algorithm based on genetic evolution and natural selection, which indicates that only the population with best properties can be chosen [2,7]. There are different methods to detect the changes in satellite imagery, which include algebra, transformation methods and advanced classification procedure. In this study, we incorporate algebra methods to identify the changes. Methods of algebra contains two image-ratio method and image differencing. According to the previous research image ratio method get higher accuracy comparing with other methods [3]. Therefore, in this article, we use these methods to detect the changes. This method has a number of benefits compared to other Change detection methods, including the reduction of atmospheric effects and deployment easier.

\section{Study Area and Data}

Due to the rapid changes in study area located in south-west of Iran, the multi temporal hyperspectral images were used to study related change. Therefore, two EO-1 Hyperion satellite images with five years interval were incorporated.

\subsection{Study Site Location}

The study area in the south-west region of Khuzestan in Iran range as the study area is considered figure-1. The geographic location is latitude and longitude $35523^{\circ} 1$. The extent of the desired region $201 \times 120$ pixels. These two datasets acquired on 2015-Apr-12 and 2010-Jun-14 for monitoring change in this area.

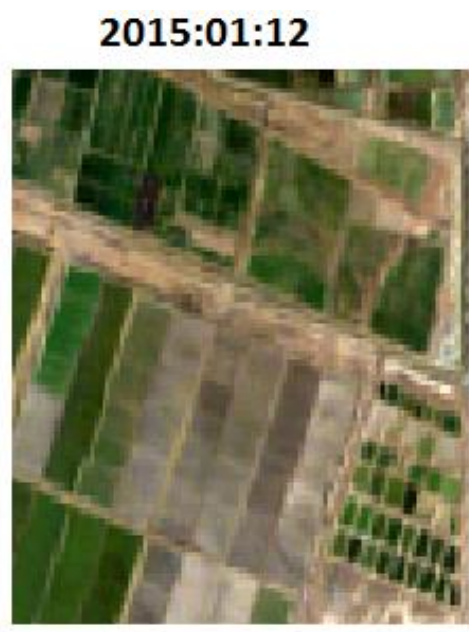

2010:06:14

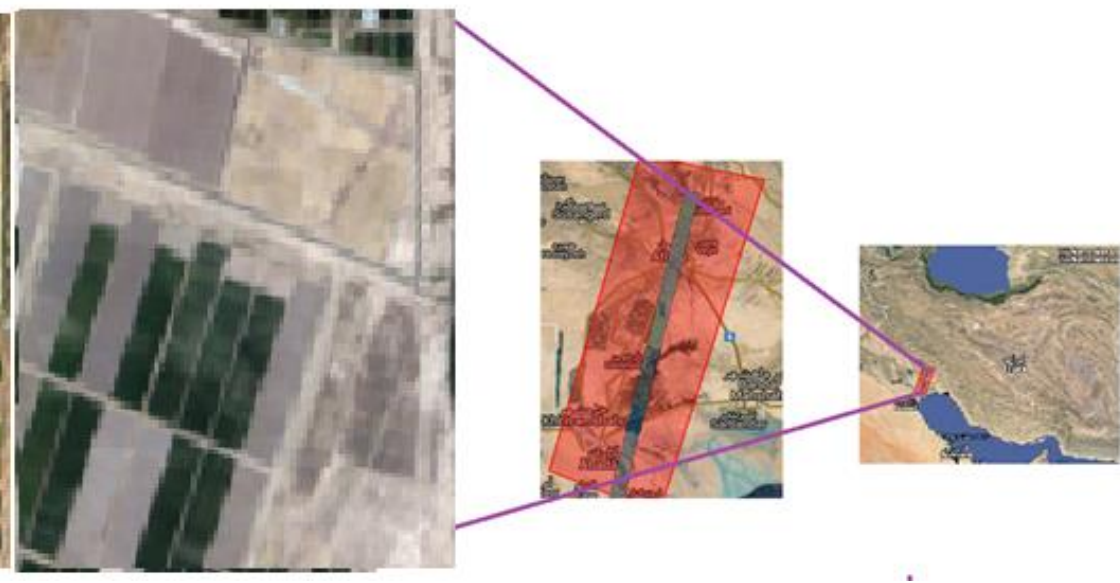

Figure-1 Study Area 


\subsection{Hyperspectral Datasets}

Most of hyperspectral sensors, acquired using aerial platform. One of most important hyperspectral satellite imagery obtains by Hyperion sensor. This sensor has 242 spectral bands wavelengths between 0.4 and 2.5 micrometers began to steadily imaging, which has a spatial resolution $30 \mathrm{~m}$ and bandwidth of $7.5 \mathrm{~km}$. Hyperion data at two separate range image by getting technology for Push broom. One of these spectra in a VNIR ranges which includes 70 band between wavelength 356-1058 nm and SWIR wavelength consists of 172 band there between wavelength $852-2577 \mathrm{~nm}$ [1].

\section{Preprocessing Hyperion of datasets}

For extracting change map using our procedure, it is necessary to do series of preprocessing that discussed below.

\subsection{Omitting noisy and zero value bands}

Among 242 bands of Hyperion imagery, there are 42 bands with zero values that these bands were include (bands 1-7, 58-76, 225242) that we remove from our imagery. Also, among the 198 bands 4 bands including, 56, 57, 77 and 78 double wide bands in the range of overlap and so noisy that we removed them from our datasets. Finally, we have 176 bands.

\subsection{De-striping bands}

Most of Hyperion bands suffer from striping. General solution to remove this error the exact offset and gain detailed knowledge related to our desired. Therefore, in this study the Hyperion-tool used as de-striping processing software.

\subsection{De-smiling}

There are a range of curvature hyperspectral error in the data center wavelength due to the change in the form of a template. To remove the effect of the application ENVI cross track illumination correction module is used.

\subsection{Convert DN to Radiance}

To achieve Radiance from digital number (DN) bands with index of 1-70 used Eq. 1.

$$
\operatorname{Rad}_{i}=D N_{i} / 40.0 . i=1: 70
$$

In addition, for SWIR range that includes the band from 71 to 242 we use Eq. 2 .

$$
\operatorname{Rad}_{i}=D N_{i} / 80.0 . i=71: 242
$$

\subsection{Atmospheric correction}

The main section of each change detection algorithm is correcting atmospheric effects for better comparing each scene of multitemporal images. In this regards, in this study, we used the Flash module for sensor Hyperion that it was in ENVI software.

\section{Purpose Methods}

In proposed method, all the bands corresponding to the first time band corresponding to the second time, according to Eq. 3.

$$
\operatorname{Ratio}_{i}=\operatorname{Rad}_{i_{T 1}} /\left(\operatorname{Rad}_{i_{T 2}}+1\right)
$$

Then each of the either of these bands are merged together. The process of integration control by a threshold value. Therefore, noise as change does not consider. Finally, a map change created. In order to select best suitable bands, form multi-temporal hyperspectral images, we used genetic algorithm (GA) for band selection. In this regards, defining fitness function, binary chromosome and other parameter is inevitable. In addition, each of the crowd with this map by comparing spectral angel mapper (SAM) function. Then begin GA that it has a number of elements, which includes the search space, gene, chromosome, the initial population, to generate the generation that includes the 
following steps (Figure-2): the formation of the initial population, evaluation fitness function and fusion/merging bands. In this process an initial population (initial solution) to solve the problem of creating. That the answers in the field as a chromosome display feeds that this chromosome is composed of a number of genes, and each gene represents a band. You can see GA parameters in Table1. Then a subset of the randomly selected respondent. In figure 2, a flowchart of proposed method has been illustrated.

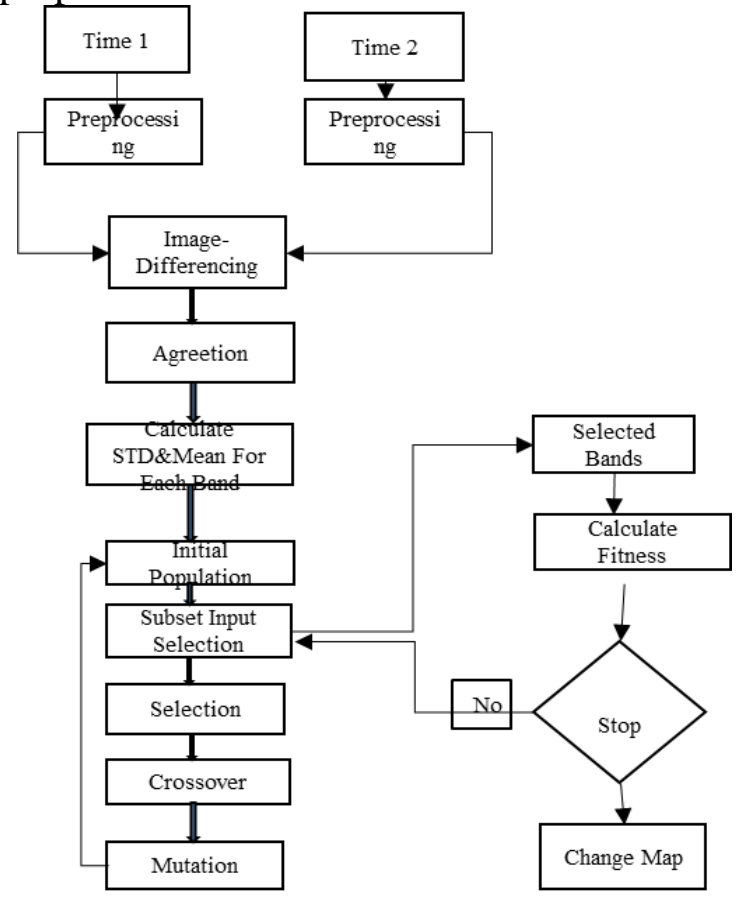

Figure (2)-flow chart of the GA change detection method.

By incorporating this procedure, the best and suitable correspond bands from both old and new hyperspectral images are selected.

Table (1)-Parameters for GA

\begin{tabular}{|l|c|}
\hline Size Initial Population & 100 \\
\hline Length of Chromosome & 176 \\
\hline Rate of Crossover & 0.8 \\
\hline Rate of Mutation & 0.01 \\
\hline Crossover & Two point \\
\hline Selection & Roulette-Wheel \\
\hline Number of Iterations & 50 \\
\hline
\end{tabular}

For better evaluating performance of our procedure the best and average output of each runs of GA illustrated in figure 3.

In addition, for the final evaluation of the accuracy of change map with respect to reduce bands by calculating the kappa coefficient between change map and index extracted from GA procedures.

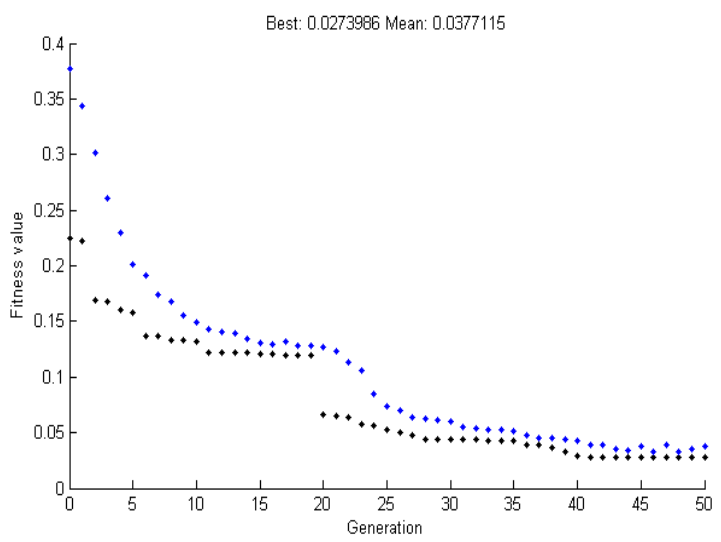

Figure (3)-Performance of GA runs for selecting best index of bands.

\section{Experiment and result}

After the implementation of the proposed method and production of results, assessment is integral part of our procedures. In this regards, we calculate the kappa coefficient for our result that nearly 0.79 . The output change map result is shown in figure 4.

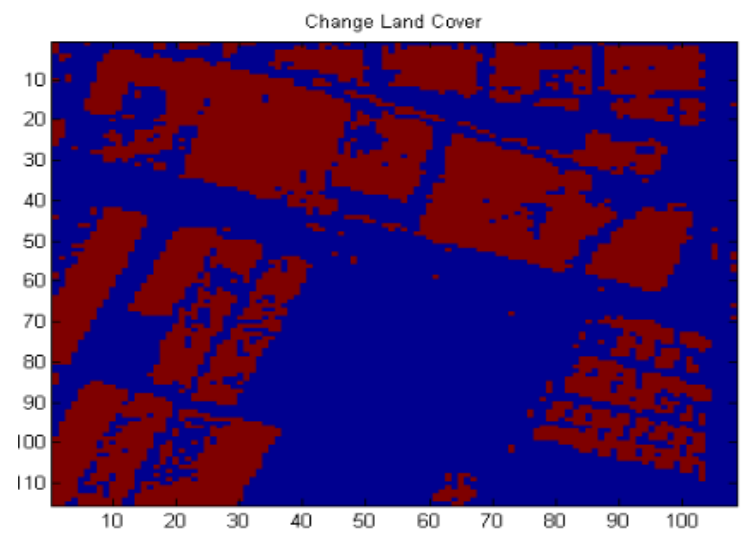

Figure (4)- The output change map result. 
Also, suitable and appropriate indies of bands from both old and new Hyperion hyperspectral images are shown in Table 2.

Table (2)- Index of best suitable bands for change map analysis

$10,11,12,13,14,16,18,19,20,21,22,24,25,26,27,28,29$ $33,34,35,36,38,39,40,41,42,44,45,46,47,48,49,55,76$ $78,79,80,81,82,83,88,90,91,96,97,98,99,100,103,104$, $105,106,107,109,110,111,113,114,115,117,119,120$, $127,132,133,134,135,136,137,138,139,141,146,148$, $149,151,153,154,155,158,160,164,165,181,182,183$, $188,191,196,198,203,204,210,213,215,217,220$

Genetic algorithm method suitable for feature selection. It is also a simple way to implement image ratio change detection methods at the same time. This procedure automatically extracts change map and requires no training data shows the changes for us.

\section{Conclusion}

In this study, we proposed procedures for selecting bands/features using GA in order to extract change map. One most benefit of this method is automatically creating changes map using multi-temporal Hyperion hyperspectral scenes. Also, accuracy of our method showed good degree of consistency to create change map (near 0.79 ).

\section{Reference}

[1] Richard Beck, etc. EO-1 User Guide (Version 2.3). 2003
[2]. C. Huang and C. Wang, "A GA-based feature selection and parameters optimization for support vector machines," Expert systems with applications, vol. 31, pp. 231-240, 2006.

[3] Zhang Shaoqing,Xu Lu,2008, “THE COMPARATIVE STUDY OF THREE METHODS OF REMOTE SENSING IMAGE CHANGE DETECTION", The International Archives of the Photogrammetry, Remote Sensing and Spatial Information Sciences.

[4] R. D. Johnson \& E. S. Kasischke, “

DETECTION OF LAND COVER CHANGES USING MULTI-TEMPORAL SATELLITE IMAGERY", The International Archives of the Photogrammetry, Remote Sensing and Spatial Information Sciences. Vol. XXXVII. Part B7. Beijing 2008

[5] J.-F. MAS 199, "Monitoring land-cover changes: a comparison of change detection techniques", int. j. remote sensing, 1999, vol. 20 , no. $1,139 \pm 152$

[6] C. Chang, "2007 Hyperspectral data exploitation theory and applications"

[7]. L. Zhuo, J. Zheng, F. Wang, X. Li, B. Ai, and J. Qian, "A genetic algorithm based wrapper feature selection method for classification of hyperspectral images using support vector machine," The International Archives of the Photogrammetry, Remote Sensing and Spatial Information Sciences, vol. 37, pp. 397-402, 2008. 